\title{
PSICOLOGIA
}

\section{A CORRELACÃO EXISTENTE ENTRE O ESTRESSE NO AMBIENTE DE TRABALHO E DOENÇAS PSICOSSOMÁTICAS}

\author{
DOI: http://dx.doi.org/10.31072/rcf.v8i2.552
}

\section{THE EXISTING CORRELATION BETWEEN STRESS IN THE WORK ENVIRONMENT AND PSYCHOSOMATIC DISEASES}

Jéssica Oliveira da Silva'; Sara Kaliana de Almeida Ferreira²; Sara Ferreira Silva ${ }^{3}$; Gésica Borges Bergamini ${ }^{4}$; Evelin Samuelsson ${ }^{5}$; Cristielli Joner6; Luiz Fernando Schneider ${ }^{7}$; Pérsia Regina Menz ${ }^{8}$.

RESUMO: O estresse é uma consequência composta por fatores físicos e psicológicos, é um fenômeno complexo que ocasiona varias doenças psicossomáticas que são reações no corpo decorrentes de fatores como o estresse. O estresse por sua vez pode ter maior presença em trabalhadores. Objetivos: Este trabalho tem como objetivo apresentar como se dá o processo de adoecimento no ambiente de trabalho, indicando as possíveis causas que levaram ao adoecimento, identificando sintomas do estresse que são potenciais no surgimento de doenças psicossomáticas, ressaltando a importância de ações preventivas. Métodos: Para isso realizou-se uma pesquisa bibliográfica, realizando um levantamento de documentos como artigos, teses e livros, utilizando-se descritores como: trabalho, estresse, doenças psicossomáticas. Resultados/Discussão: A importância que o trabalho tem na vida das pessoas pelo fator de sobrevivência e social, e por isso, passa a ter relação direta com as condições de saúde física e mental do trabalhador, pois tem sua relação de prazer e sofrimento afetada, o que pode levar a um adoecimento físico e/ou psíquico. Considerações: É necessário que as empresas/organizações observem e compreendam a importância de ações que previnam o estresse e possíveis doenças relacionadas ao trabalho.

Palavras-chave: Trabalho. Estresse. Doenças psicossomáticas.

\footnotetext{
1 Graduanda em Psicologia pela Faculdade de Educação e Meio Ambiente - FAEMA. E-mail: kha.oliveira@hotmail.com. ORCID: https://orcid.org/0000-0002-0672-8380;

2 Graduanda em Psicologia pela Faculdade de Educação e Meio Ambiente - FAEMA. E-mail: sarakaliana@gmail.com. ORCID: https://orcid.org/0000-0001-6359-9944;

3 Graduanda em Psicologia pela Faculdade de Educação e Meio Ambiente - FAEMA. E-mail: sarasilvaff4@gmail.com. ORCID: https://orcid.org/0000-0002-3954-5652;

${ }^{4}$ Mestra, Psicóloga e Orientadora desta pesquisa. Faculdade de Educação e Meio Ambiente - FAEMA. Email: gpensemagro@hotmail.com. ORCID: https://orcid.org/0000-0003-0598-5366;

5 Mestra, Bióloga e Colaboradora desta pesquisa. E-mail: evelin.samuelsson@faema.edu.br. ORCID: https://orcid.org/0000-0002-0508-2709;

6 Especialista, Fisioterapeuta e Colaboradora desta pesquisa. E-mail: cristielle.joner@faema.edu.br. ORCID: https://orcid.org/0000-0002-7476-667X;

7 Especialista, Fisioterapeuta e Colaborador desta pesquisa. E-mail: luiz.schneider@faema.edu.br. ORCID: https://orcid.org/0000-0002-7945-2581;

${ }^{8}$ Mestra, Fisioterapeuta e Colaboradora desta pesquisa. Faculdade de Educação e Meio Ambiente - FAEMA. E-mail: persia.menz@faema.edu.br. ORCID: https://orcid.org/0000-0002-1052-6650.
} 
ABSTRACT: Stress is a consequence composed of physical and psychological factors, is a complex phenomenon that causes several psychosomatic diseases that are reactions in the body due to factors such as stress. Stress in turn may have a greater presence in workers. Objectives: This study aims to present how the process of illness in the work environment, indicating the possible causes that lead to illness, identifying symptoms of stress that are potential in the emergence of psychosomatic diseases, emphasizing the importance of preventive actions. Methods: For this purpose, a bibliographic research was carried out, carrying out a survey of documents such as articles, theses and books, using descriptors such as: work, stress, psychosomatic diseases. Results / Discussion: The importance that the work has in the life of the people by the survival and social factor, and therefore, has a direct relation with the physical and mental health conditions of the worker, as it has its relation of pleasure and suffering affected, which can lead to physical and / or psychic illness. Considerations: It is necessary for companies / organizations to observe and understand the importance of actions that prevent stress and possible work-related illnesses.

Keywords: Work. Stress. Psychosomatic diseases.

\section{INTRODUÇÃO}

O trabalho desempenha um papel na sociedade e tem grande importância na vida de cada trabalhador. Trabalhar pode ser tanto uma fonte de realizações e de prazer como pode causar sofrimento ao trabalhador. Acontece que, dependendo das condições em que 0 trabalho é realizado, ele pode causar sofrimento, adoecimento e até mesmo a morte. (1)

Para Dejours (2) o trabalho é mais do que a venda da força de trabalho pela remuneração. É uma atividade que se dirige ao outro, pois, trabalha-se para alguém, para um chefe, para nossos funcionários, colegas. Existe também uma remuneração social pelo trabalho, na medida em que ele permite ao trabalhador sentir-se pertencente a grupos, e lhe proporciona condições de possuir direitos sociais ${ }^{(3)}$.

Desse modo, o trabalho ocupando lugar tão relevante na vida das pessoas, passa a ter relação direta com as condições de saúde tanto física quanto mental. O trabalho, por ser um dos fatores centrais na construção da subjetividade humana, afeta a relação de prazer e sofrimento no trabalho que, por sua vez, pode se transformar em adoecimento físico e/ou psíquico; daí sua estreita ligação com a saúde (1).

As doenças do trabalho referem-se a um conjunto de danos ou agravos que incidem sobre a saúde dos trabalhadores, causados, desencadeados ou agravados por fatores de risco presentes nos locais de trabalho. Manifestam-se de forma lenta, insidiosa, podendo levar anos, às vezes 
até mais de 20 anos, para manifestarem o que, na prática, tem demonstrado ser um fator que dificulta no estabelecimento da relação entre uma doença sob investigação e o trabalho ${ }^{(4)}$.

De acordo com o Anuário da Saúde do Trabalhador (5), em comparação a 2004, os afastamentos por doença ocupacional, cresceram $9,4 \%$, e chegaram a quase 181 mil casos em 2014. Com isso, este trabalho tem como objetivo apresentar como se dá o processo de adoecimento no ambiente de trabalho, indicando as possíveis causas que levaram ao adoecimento, identificando sintomas do estresse que são potenciais no surgimento de doenças psicossomáticas, ressaltando a importância de ações preventivas. Através do levantamento bibliográfico de artigos, teses, e outros que foram selecionados priorizando o mesmo objetivo do presente artigo. Em especial, uma tese de pesquisa exploratória com estudo de caso na qual se propôs investigar os servidores de uma universidade pública e que, para isso se utilizou de método quantitativo.

Portanto, compreende-se que há uma correlação entre 0 adoecimento no trabalho, o estresse e o funcionamento organizacional. Porém, a organização pode realizar ações preventivas através dos diversos setores organizacionais evidenciando um bom clima organizacional e ambiente propicio ao desenvolvimento produtivo e saudável.

\section{METODOLOGIA}

Este estudo é resultado de uma pesquisa bibliográfica, onde foi realizado um levantamento de documentos como artigos, teses e livros. A busca literária ocorreu no período de outubro de 2017. Utilizaram-se os seguintes descritores: trabalho, estresse, doenças psicossomáticas. Os bancos de dados utilizados foram: Scielo; BVS; Google Acadêmicos. Para seleção dos artigos que foram utilizados nesta descrição foi utilizado como critério de exclusão àqueles documentos que não tinham os descritores em suas palavras-chave. Ao todo se utilizou 28 obras.

\section{ESTRESSE}

O estresse é um fenômeno complexo, com uma multiplicidade de conceitos que quando, colocados à prova, demonstram fragilidade de toda ordem. Estresse pode ser definido como "uma consequência direta dos persistentes esforços adaptativos da pessoa à sua situação existencial" (6). Situação que é marcada por muitas exigências devido às incansáveis mudanças da vida moderna imposta às pessoas e que, como consequência, cobram ajustamentos constantes. Isto expõe as pessoas a 
situações de conflitos frequentes que as desestabilizam emocionalmente, caracterizadas por ansiedades $\mathrm{e}$ angústias, dentre outras ${ }^{(7)}$.

O estresse é uma reação complexa composta por aspectos físicos e psicológicos, diante dos quais, frequentemente, trabalhadores têm reações negativas posto aos distúrbios causados. Perante a escassez dos meios de enfrentamento e o prolongamento de suas causas, o estresse pode avançar para um quadro de maior gravidade, tornando-se o corpo vulnerável a diversas doenças, inclusive as doenças psicossomáticas ${ }^{(8)}$.

A presença do estresse no ambiente de trabalho, não é tarefa fácil de avaliar. Pois muitas vezes agentes estressores são passados despercebidos e por isso não são encarados devidamente. O estresse é o representante emocional da ansiedade. A ansiedade interfere na atenção ou na realização de tarefas $(7,8)$. Segundo Gonçalves (8), "o estresse é um inimigo silencioso e perigoso".

Couto ${ }^{(9)}$ denomina o estresse como uma degradação anormal do organismo humano, gerando a diminuição na sua capacidade de trabalho ocasionada pela inaptidão que o individuo não possui de admitir e ultrapassar todas as cobranças psíquicas da vida e conciliá-las. A degradação anormal do organismo humano é provocada por uma inquietude arraigada que é característica da contemporaneidade. Ressalta-se ainda que as pessoas mais acometidas pelo estresse são aquelas que não obtêm situações como relaxar e descontrair mesmo que seja do seu desejo.

$O$ estresse em especial tem ganhado milhares de estudos na contemporaneidade e chamado à atenção das organizações em relação à produtividade de seus colaboradores. O estresse afeta não somente os trabalhadores, mas a organização e a sociedade como um todo o que pode ser diminuído através de ações na organização.

Com um olhar fisiológico Pereira ${ }^{(10)}$ conceitua 0 estresse como sendo 0 responsável em incitar no individuo alterações que preparam o organismo a superarem e/ou enfrentarem as raízes de pressão excessiva na qual o mesmo é sujeitado.

\section{DOENÇAS PSICOSSOMÁTICAS}

O termo "psicossomático" foi utilizado pela primeira vez em 1918 pelo psiquiatra alemão J. C. Heinroth, quando escreveu um artigo onde ressaltava a importância e a influência das paixões sobre a tuberculose e o câncer (11). Volich (12) afirma que a psicossomática, herdeira das 
correntes que concebem a unidade corpomente, busca compreender a existência humana, a saúde e a doença segundo essa visão integrada. $E$ tem a intenção de compreender uma visão de integralidade do homem, ou seja, sua totalidade, um complexo mente-corpo em interação com um contexto social (13).

A psicossomática $($ psico $=$ mente, soma $=$ corpo) é considerada como uma integração entre os processos biológicos, mentais e físicos, observável ou não, que apresentam reações em todo o corpo ${ }^{(14)}$. A psicossomática representa um problema emocional que um indivíduo possa ter. A partir desse problema emocional 0 indivíduo passa a sentir diversos sintomas físicos, sendo estes por sua vez de extrema ordem emocional. A somatização que são as disfunções físicas, visíveis ou não, ocorridas na mente, faz com que o corpo sinta diversos sintomas, tais como dor, mal-estar e doenças ${ }^{(15) .}$

Segundo Freire ${ }^{(16)}$, o termo psicossomático, que é compreendido como a relação do corpo e mente, ou seja, da interdependência dos aspectos biológicos e psicológicos, também pode ser utilizado para tratar da correlação entre trabalho e saúde mental. O estresse é um dos precursores que contribuem para 0 surgimento de milhares de doenças psicossomáticas como a psoríase, envelhecimento precoce. São também percebidas através de afastamento médico gerando um alto custo para as empresas, havendo dessa maneira a necessidade de identificar quais são os agentes estressores nas organizações.

5 RELAÇÕES ENTRE O ESTRESSE NO AMBIENTE DE TRABALHO E DOENÇAS PSICOSSOMÁTICAS

Pode-se dizer que as doenças psicossomáticas vêm da ausência de qualidade de vida no trabalho. As relações que se estabelecem entre essas doenças psicossomáticas e o estresse no ambiente de trabalho se dão pelo motivo de não existir uma qualidade social, afetiva, profissional e emocional no dia-a-dia dos funcionários dentro da organização ${ }^{(7,10,11)}$.

Para Freire (16), os aspectos emocionais são compreendidos como fonte de alimento do corpo físico, que na impossibilidade de se expressar, pode comprometer o seu equilíbrio. Wolff, um dos fundadores e presidente da Sociedade Americana de Psicossomática, em 1952, demonstrou que os distúrbios da relação do homem com seu ambiente físico e psicossocial podem gerar emoções desprazerosas e desencadear diversas reações, inclusive doenças ${ }^{(17)}$.

O indivíduo vai ter sua capacidade comprometida a partir do momento em que se depara com situações que lhe causam 
sintomas com uma frequência constante. Nem todas as pessoas compreendem o que aquele indivíduo sente. Se todas as vezes que este sentir algo, for à procura de um médico, e não tiver nada, ele passara a ser visto pelos demais como um oportunista, podendo gerar mais sofrimento a esse indivíduo ${ }^{(9,10)}$.

Um passo importante para se entender as doenças psicossomáticas, foi entender o estudo da pessoa como ser histórico, constituído por três subsistemas: corpo mente e social. Desta forma, o corpo passa a ser entendido como expressão do constante contato com o mundo externo (18). Dando uma configuração de um movimento médico verdadeiramente aplicado à promoção da saúde.

É fundamental a insistência de se levar em consideração a totalidade do ser humano $\mathrm{e}$ das circunstâncias que $\mathrm{o}$ rodeiam para termos uma compreensão mais ampla dos processos de adoecer. A totalidade surge quando se leva em conta a pessoa e não a doença (18).

Para Freire (19), o ser humano é um todo que envolve aspectos biológicos e socioculturais e seu bem-estar está relacionado à suas relações com os grupos os quais este está inserido. Esta influência histórico-social se faz de acordo com o grupo social ao qual eles pertencem, e que reforçará ou punirá os seus comportamentos ${ }^{(18)}$.

Daí a tendência atual da Psicossomática de compreender os processos de adoecer, não como um evento casual na vida de uma pessoa, mas sim representando a resposta de um sistema, de uma pessoa que vive em uma sociedade (18). É como é de extrema importância que seja ampliada a capacidade de entendimento sobre o comportamento das pessoas e onde são influenciados socialmente.

As organizações se tornam cada vez mais seletiva e criteriosa quando se relaciona com a lucratividade e o desempenho de seus colaboradores, intensificando o desejo por profissionais com habilidades e aptidões que façam a diferença. A qualidade de vida $e$ as motivações desses colaboradores nesse cenário são afetadas refletindo direta ou indiretamente na qualidade dos serviços prestados na organização a qual o mesmo possui vínculo empregatício $(15,17,20)$.

$O$ estresse surge diante dos fatos citados como uma obstrução rompendo dessa maneira o equilíbrio do indivíduo. Jorge (20) elucida que o estresse tem afetado um número cada vez maior de trabalhadores e as consequências e os sintomas que esse distúrbio traz vai de uma dor no ombro até o infarto do 
miocárdio. O estresse "ainda" não é reconhecido como uma instância patológica, todavia é uma disfunção fisiológica, onde acarretam vestígios que induzem a doenças e incômodos provocando sofrimento.

Vários fatores contribuem para que haja o rompimento do equilíbrio do individuo afetando dessa forma a qualidade de vida e do trabalho como, por exemplo, o trânsito até chegar à empresa, em grandes metrópoles existe a necessidade de pegar 3,4 ou mais conduções para chegar no horário estabelecido pela empresa. Lipp afirmam que a sobrecarga de trabalho e na família, a relação com o patrão, a cobrança, falta de compreensão, de união e cooperação com a equipe de trabalho, o salário que não corresponde as suas necessidades ou ao trabalho que desempenha, falta de motivação da empresa e a automotivação e o meio social são outros motivos causadores do estresse.

A Síndrome de Burnout que é um tipo de estresse ocupacional, ou seja, um esgotamento profissional que tem como principal característica tensão emocional e estresse crônico provocado por condições físicas de trabalho, psicológicas e emocionais. Marras \& Veloso (22) consideram que os indivíduos vivem em realidades construídas socialmente e que, por força das circunstâncias sociais, e que na maioria das vezes o individuo não sabe reagir da maneira correta aos estresses do dia-a-dia, o indivíduo passa a experimentar situações estressantes no ambiente de trabalho, até mesmo por ser uma necessidade estar ali. Todavia existem, indivíduos que buscam de diversas maneiras minimizar impactos do estresse.

\section{RESULTADOS E DISCUSSÕES}

Houve muitas mudanças no processo de trabalho com a evolução tecnológica, as pessoas tiveram que se adaptar aos novos maquinários, tiveram a necessidade de assumir várias funções e turnos diferentes para que pudessem alcançar o ritmo das máquinas e não ser superado por estas, e isso tudo fez com que ocorresse um aumento da competitividade dentro das organizações. Toda essa pressão no trabalho gerar aumento de ansiedade, da tensão e do sentimento de insegurança; esses fatores contribuem para o sofrimento psíquico do trabalhador, Simões (23). Tem um lado bom em todo esse avanço tecnológico que permite um menor esforço braçal dos trabalhadores e com isso um aumento em sua expectativa e qualidade de vida. Contudo, por outro lado, essa busca acelerada por esse desenvolvimento tecnológico leva à um aumento da possibilidade de sofrimento, 
doenças e mortes por conta do estilo de vida que é imposto pela sociedade contemporânea.

É importante que se tenha uma boa configuração nas relações entre trabalhador e o ambiente de trabalho. Nos casos onde essa relação não tem um bom desenvolvimento, possibilita supor que esse modelo de produção não é favorável para satisfação e equilíbrio psicossomático do trabalhador, pois, a maneira como ele recorre ao trabalho e o modelo de produção que é exigido pela organização, vão exigir desse trabalhador um posicionamento que não permita com que esse se envolva efetivamente com seu trabalho. O ritmo acelerado de produção da organização gera a sobrecarga de serviço e, como consequência, o ambiente do trabalho exerce sobre o funcionário uma sobrecarga excessiva (23). E isso vai fazer com que o trabalhador se sinta muito desestimulado para participar da gestão de onde trabalha, fazendo com que este perca sua capacidade de perceber e seus sentimentos. Se esse trabalhador não consegue ter uma percepção dos seus processos psíquicos, é bem improvável que ele consiga se posicionar frente seus problemas.

Para Dejours e Abdoucheli (24) o conflito entre organização do trabalho e funcionamento psíquico pôde ser reconhecido como fonte de sofrimento, ao mesmo tempo como chave de sua possibilidade de análise. Mas o sofrimento suscita estratégias defensivas. A descoberta empírica mais surpreendente foi a das estratégias defensivas construídas, organizadas e gerenciadas coletivamente. Para eles essas defesas levam à modificação, transformação e, em geral, à eufemização da percepção que os trabalhadores têm da realidade que os faz sofrer. Tudo se passa como se, por falta de poder vencer a rigidez de certas pressões organizacionais irredutíveis, os trabalhadores conseguissem, graças a suas defesas, minimizar a percepção que eles têm dessas pressões, fontes de sofrimento. Essas estratégias, em geral, funcionam por um retorno da relação subjetiva com as pressões patogênicas.

De vítimas passivas, os trabalhadores colocam-se na posição de agentes ativos de um desafio, de uma atitude provocadora ou de uma minimização diante da dita pressão patogênica. A operação é estritamente mental, já que ela geralmente não modifica realidade da pressão patogênica. Com relação ao seu grau de generalidade, parece que, na medida em que se desenvolviam as pesquisas, as estratégias coletivas de defesa estavam bem difundidas, mas não se pode afirmar que 
elas estejam presentes em todas as situações de trabalho ${ }^{(24)}$.

Dejours e Abdoucheli (24) falam também da relação entre estratégia coletiva de defesa e alienação. Ele levanta a questão, se ao transformar a percepção da realidade, as estratégias coletivas de defesa não estariam arriscando-se a enganar os trabalhadores, a mascarar 0 sofrimento e a perturbar a ação ou luta contra as pressões patogênicas da organização do trabalho? Eles assumem a existência desse risco, e colocam que se as estratégias defensivas são necessárias para a continuação do trabalho e adaptação às pressões para evitar a loucura, em contrapartida elas contribuem para estabilizar a relação subjetiva com a organização do trabalho, no estado em que ela se encontra e a alimentar uma resistência à mudança. Quando os trabalhadores conseguem estruturar estas defesas, eles hesitam em questioná-las. Deve-se considerar, na investigação, que o sofrimento que se pretende analisar não será obtido senão através de estratégias defensivas, que, por sua vez, transformaram, profundamente, a expressão deste sofrimento. E por fim, trazem que se lembrarmos que cada coletivo de trabalho constrói suas próprias estratégias coletivas de defesa, poderá estudar como elas resultam, às vezes, em impasses que contribuem precisamente para radicalizá-las em ideologias defensivas ${ }^{(24)}$.

Todo esse impacto de adoecimento causado pelas relações de trabalho na saúde do trabalhador exige novas perspectivas quanto ao binômio homemtrabalho que gerem intervenções adequadas, principalmente na esfera preventiva.

Logo, desenvolver programas que reduzam o estresse provocará saúde e qualidade de vida no contexto organizacional, impelindo com isso 0 desenvolvimento humano e, consequentemente, a eficiência e o crescimento das organizações (25).

Dentre as medidas que podem ser tomadas para se prevenir o adoecimento dos trabalhadores está a criação de programas voltados à qualidade de vida, que proporcionem o alívio das tensões do dia-a-dia, o lazer. Um exemplo disso é o Programa de Qualidade de Vida - PQV, do qual, tendo já participado, Gonçalves (8) percebeu que muitos servidores não participam. Segundo ela isso se deve às muitas responsabilidades do serviço, às obrigações familiares, que não permitem o relaxamento.

Propomos também o olhar mais atencioso por parte da chefia e/ou do trabalhador para os agentes estressores. 
Que, diante das evidencias apresentadas sobre a importância desse assunto haja uma postura interventiva dos dirigentes.

Outra proposta é o acompanhamento e tratamento do trabalhador, desde o início do aparecimento de sintomas e situações de estresse, pois, com certeza, tais medidas proporcionariam a diminuição dos afastamentos e outras ocorrências.

Por causa do estresse advindo da rotina dos trabalhadores, a exemplo de Pereira ${ }^{(26)}$, recomenda:

- Conscientização por parte de equipes preparadas para transmitir informações sobre saúde do trabalhador;

- Promover a valorização e motivação dos trabalhadores através de palestras e outras ações que abordem assuntos direcionados aos mesmos;

- Oferecer atendimento aos trabalhadores que atendam as queixas relacionadas ao estresse e proporcionem saúde.

Contribuições relevantes a sociedade seria a disseminação em massa de informações sobre a temática apresentada neste trabalho, que alcancem trabalhadores de diversos setores e ramos como também estudantes universitários. Inclui-se a sugestão da continuidade de estudos que abordem essa temática relacionando-a aos universitários.
De acordo com Spector (27), as condições físicas de trabalho tendem a ter efeitos físicos e psicológicos diretos sobre o trabalhador. Esses efeitos físicos podem ser imediatos, no caso de algum acidente onde o funcionário saia ferido ou podem desenvolver doenças ou lesões com a exposição a uma condição danosa por um longo tempo, como substâncias tóxicas ou ruídos excessivos. Os efeitos psicológicos estariam ligados a doenças e lesões que podem vir a se associar a algum nível de sofrimento psíquico, trauma ou angustia (28).

As Lesões por Esforço Repetitivo (LER) é uma das lesões que podem se desenvolver no indivíduo quando este executa ações com movimentos repetitivos. Há os casos onde o trabalhador tem que levantar cargas pesadas, que podem resultar em lesões agudas. Esses dois tipos de lesões constituem a forma de lesão muscoesquelética, e essas lesões ainda podem ter uma relação psicológica, que está ligada ao estresse no trabalho. Portanto, as pessoas que tiverem pouca autonomia e controle no trabalho, que não estiverem satisfeitas ou se sentirem-se deprimidas ou ansiosas, estarão mais propensas a esse tipo de lesão (27)

Outro fator prejudicial para a saúde do trabalhador é o horário de trabalho, 
pessoas que trabalham de noite desenvolvem distúrbios de sono, problemas no sistema digestivo, entre outros, além de problemas sociais, pois ter quer trabalhar durante a noite e dormir durante o dia pode fazer com que a pessoa de afaste da família e amigos $(27,28)$. Ainda com relação a horário de trabalho, longos expedientes também são muito prejudiciais para o sujeito, esse trabalhador ficará fadigado, pois trabalhando de 10 a 12 horas exige muito esforço físico e mental. Podendo também desenvolver no trabalhador problemas de sono, uso de estimulantes e consumo de álcool, entre outros problemas ${ }^{27,29)}$.

O estresse é outro fator que afeta muito a vida do trabalhador, muitos elementos podem ser estressantes no ambiente de trabalho, como conflitos com colegas, uma carga excessiva de trabalho, se comportar de uma forma que vá contra seus ideais, problemas com a política organizacional, ritmo determinado pela máquina, modelo de controle demanda. Conflitos entre trabalho e família também podem gerar um grande nível de estresse na vida de uma pessoa, muitas vezes por conta do trabalho a pessoa não tem tempo para família, pais e mães não conseguem ficar com seus filhos, ou precisam se ausentar do trabalho para levá-los ao médico, por exemplo. Essa questão pode ser mais prejudicial para as mulheres que, normalmente assumem a maior responsabilidade pelos filhos ${ }^{(27,29)}$.

Acidentes no trabalho são um importante causador de morte de pessoas na idade ativa. Tartari ${ }^{(30)}$ sugere que um acidente sofrido pelo trabalhador, estando ou não no local de trabalho, que esteja a serviço da empresa, que provoque alguma lesão corporal, algum comprometimento na capacidade de trabalho ou alguma doença ou perturbação que leve a morte, pode ser definido como acidente de trabalho. E uma grande preocupação das organizações é a prevenção de acidentes, existem várias estratégias que podem ser utilizadas, porém a melhor dependerá da situação específica de uma análise das causas dos acidentes $(2728,29,30)$.

$O$ estresse dentro e fora do trabalho pode ser um importante fator na causa de acidentes no trabalho, a forma como as pessoas se sentem referentes ao seu emprego e suas atitudes com relação à segurança também afetam no risco de acidentes e lesões no trabalho, por isso a importância de que seja construído um ambiente saudável e que transmita confiança ao trabalhador, pois pessoas satisfeitas e com sentimento de segurança com relação a seu trabalho são menos propensas a sofrer algum acidente no trabalho $(27,28,30)$. 
Portanto, podemos perceber que as relações que se estabelecem entre 0 sofrimento psíquico e os aspectos relacionados ao trabalho se caracterizam como um desafio a ser enfrentado conjuntamente. Para tanto, é necessária uma prática que não tenha meramente cunho normativo ou reducionista, mas que seja capaz de integrar aspectos da saúde e da doença dos trabalhadores com o objetivo de dar outro significado ao sofrimento vivido no mundo do trabalho, resgatar o sentido do trabalho para o trabalhador, para a organização e para a sociedade de forma a melhorar a qualidade de vida (23).

Para isso se faz necessário sinalizar a importância de diagnosticar efetivamente

\section{REFERÊNCIAS}

1. Merlo ÁRC, Bottega CG, Perez KV. Atenção ao Sofrimento e ao Adoecimento Psíquico do Trabalhador e da Trabalhadora: Cartilha para Profissionais do Sistema Único de Saúde - SUS. Porto Alegre :Evangraf; 2014. [citado em 28 de outubro de 2017]. Disponível em: https://www.forumat.net.br/at/sites/default/fi les/arq-paginas/5869.compressed.pdf.

2. Dejours C, Molinier P. O Trabalho como Enigma. In:Lancman S,Sznelwar L, organizadores. Christophe Dejours: $\mathrm{Da}$ Psicopatologia à Psicodinâmica do Trabalho. $1^{\underline{a}}$ ed. Rio de Janeiro (RJ): Fio Cruz; 2004.

\section{Dejours C. Avant-propos para a Edição} Brasileira. Addendum: Da Psicopatologia à as causas do estresse e de seu impacto nas relações de trabalho e na qualidade de vida dos trabalhadores. De forma a facilitar e direcionar objetivamente o planejamento de intervenções que promovam saúde e bem-estar a estes.

\section{CONSIDERAÇÕES FINAIS}

Dessa forma, notamos que 0 trabalho ocupa um lugar de muita importância na vida das pessoas, com isso, passa a ter relação direta com as condições de saúde física e mental do trabalhador, pois tem sua relação de prazer e sofrimento afetada, o que pode levar a um adoecimento físico e/ou psíquico. O que faz com que seja de extrema importância que seja pensado em ações que previnam essas doenças.

Psicodinâmica do Trabalho. In: Lancman S, Sznelwar L, organizadores. Christophe Dejours: $\mathrm{Da}$ Psicopatologia à Psicodinâmica do Trabalho. $1^{\text {a }}$ ed. Rio de Janeiro (RJ): Fio Cruz; 2004.

4. Ministério da Saúde. Brasília: Cadernos de Atenção Básica: Programa Saúde da Familia - № 5. Saúde do Trabalhador; 2001. [citado em 28 de outubro de 2017]. http://bvsms.saude.gov.br/bvs/publicacoes/ saude_trabalhador_cab5_2ed.pdf

5. Anuário da saúde do trabalhador / Departamento Intersindical de Estatística e Estudos Socioeconômicos. - São Paulo : DIEESE, 2016.

6. Ballone GJ. Stress. Psiq Web Psiquiatria Geral 2002. [citado em 28 de outubro de 
2017].

Disponível

em: http://www.psiqweb.med.br/cursos/stress.

7. Favassa CTA, Armiliato N,Kalinine I. Aspectos Fisiológicos e Psicológicos do Estresse. Rev de Psicologia da UnC 2005; 2(2): 84-92. [citado em 28 de outubro de 2017]. Disponível em: http://www.academia.edu/download/33838 983/Estresse_-

Aspectos_Fisiologicos_e_Psicologicos_d o_Estresse.pdf.

8. Gonçalves RA. Estresse Profissional: Estudo de Caso sobre os Principais Sintomas Apresentados pelos Servidores de uma Universidade Pública. São Carlos. Monografia [Especialização em Gestão Pública] - Universidade Federal de São Carlos; 2011. [citado em 28 de outubro de 2017]. Disponível em: http://www.progpe.ufscar.br/blog/cqa/wpcontent/uploads/MONOGRAFIA_GESTAO _PUBLICA_ROSELI_A_GONCALVES.pdf.

9. Couto HA. Stress e Qualidade de Vida do Executivo. Rio de Janeiro (RJ): Cop; 1987.

10. PereiraLZ,Zille GP. O Estresse no Trabalho: Uma Análise Teórica de seus Conceitos e suas Inter-Relações. Rev Gestão e Sociedade 2010; 4(7): 414-434. [citado em 28 de outubro de 2017]. Disponível em: http://www.gestaoesociedade.org/gestaoes ociedade/article/view/923.

11. Monteiro CS, Todaro AP. Sofrimento no Trabalho: Nem Tudo é o que Parece Ser. Contribuições da Psicodinâmica do Trabalho e da Psicossomática. Volta Redonda (RJ): Universidade Federal Fluminese; 2014. [citado em 28 de outubro de 2017].Disponível em: http://repositorio.uff.br/jspui/bitstream/1/20 38/3/2014-Administra\%C3\%A7\%C3\%A3oCHANDER\%20SANTOS\%20MONTEIRO. pdf.

12. Volich RM. Psicossomática. 6ª ed. São Paulo: Casa do Psicólogo; 2000.[citado em
28 de outubro de 2017].Disponível em: https://books.google.com.br/books?hl=pt$B R \& \mid r=\& i d=x u 98 Q g v k 3 r E C \& o i=f n d \& p g=P A$ 19\&dq=Volich+RM.+Psicossom\%C3\%A1ti ca. $+6 \%$ C2\%AA+ed.+S\%C3\%A3o+Paulo:+ Casa+do+Psic\%C3\%B3logo\%3B+2000.\&o ts $=$ OOLIEm1_rq\&sig=9Ri25UWnDQ0qVuZ xF14j0sl5oM4\#v=onepage\&q=Volich\%20R M.\%20Psicossom\%C3\%A1tica.\%206\%C2 $\%$ AA\%20ed.\%20S\%C3\%A30\%20Paulo\%3 A\%20Casa\%20do\%20Psic\%C3\%B3logo\% $3 B \% 202000 . \& f=$ false.

13. Lipowiski ZJ. O que a Palavra "psicossomática" realmente significa? Uma investigação histórica e semântica. Medicina Psicossomática 1984; 46 (2): 153-171.

14. França ACL. Psicologia do Trabalho: Psicossomática, Valores e Práticas Organizacionais. São Paulo (SP): Saraiva; 2008.

15. Carvalho ML. Qualidade de Vida no Trabalho Versus Condições Psicossomáticas Advindas do Mercado de Trabalho. Rev REGRAD 2016; 9(1): 67-84. [citado em 28 de outubro de 2017]. Disponível em: http://revista.univem.edu.br/REGRAD/articl e/view/1321.

16. Freire CA. O Corpo Reflete o seu Drama: Somatodrama como Abordagem Psicossomática. São Paulo: Agora; 2000. [citado em 28 de outubro de 2017]. Disponível em: https://books.google.com.br/books?hl=pt$B R \& \mid r=\& i d=S L s \_7 k C h Y 3 A C \& o i=f n d \& p g=P$ $\mathrm{A} 4 \& \mathrm{dq}=$ Freire $+\mathrm{CA} .+\mathrm{O}+\mathrm{Corpo}+$ Reflete $+0+\mathrm{S}$ eu+Drama:+Somatodrama+como+Aborda gem+Psicossom\%C3\%A1tica.+S\%C3\%A3 o+Paulo:+Agora\%3B+2000.\&ots=kFU02yo Ytn\&sig=5Pb1FR9gPkni4YLt6JaKmyeJeh $\mathrm{w} \# \mathrm{v}=$ onepage $\& \mathrm{q} \& \mathrm{f}=$ false .

17. França ACL, Rodrigues LR. Stress e Trabalho: Uma Abordagem Psicossomática. $4^{\underline{a}}$ ed. São Paulo (SP): Atlas; 2005. 
18. Filho JM, Burd M, colaboradores. Psicossomática Hoje. 2 ${ }^{\underline{a}}$ ed. Porto Alegre (RS): Artmed; 2010.[citado em 28 de outubro de 2017]. Disponível em: https://books.google.com.br/books?hl=pt$B R \& \mid r=\& i d=03 A s 2 a s V R w Y C \& o i=f n d \& p g=P$ R4\&dq=MELLO+FILHO,+J\%3B+et+al.+Psi cossom\%C3\%A1tica+Hoje.+Porto+Alegre $+(\mathrm{RS}):+$ Artmed\%3B+2010.\&ots=NSqlilNaK b\&sig=tu7csPrm11CF5QZ1xtWkXQ7pNhA $\# \mathrm{v}=$ onepage \& $\mathrm{q} \& \mathrm{f}=\mathrm{false}$.

19. Freire $\mathrm{G}$. Médicos Doentes e Contextos Sociais: Uma Abordagem Sociológica. Rio de Janeiro (RJ): Globo; 1983.

20. Jorge IMP. Doenças Psicossomáticas Relacionadas ao Trabalho - Estudo de Caso. Florianópolis. Dissertação [Mestrado em Engenharia de Produção] Universidade Federal de Santa Catarina; 2004. [citado em 28 de outubro de 2017]. Disponível em: https://repositorio.ufsc.br/bitstream/handle/ 123456789/101557/223668.pdf?sequence $=1$ \&isAllowed $=y$

21. Lipp MEN. Stress no Trabalho: Implicações para a Pessoa e para a Empresa. In: Sobrinho FPN, Nassaralla I. Pedagogia Institucional: Fatores Humanos nasOrganizações. Rio de Janeiro (RJ): Zit Editora; 2005.

22. Marras JP, Veloso HM. Estresse Ocupacional. Rio de Janeiro (RJ): Elsevier; 2012.

23. Simões FIW, Hashimoto F. Adoecimento no Trabalho: Um Estudo de Caso. Rev Laborativa 2013; 2(2): 7385.[citado em 28 de outubro de 2017]. Disponível em: http://200.145.6.204/index.php/rlaborativa/ article/view/937/pdf.

24. Dejours C, Abdoucheli E, Jayet C, Betiol MIS. Psicodinâmica do Trabalho: Contribuição da Escola Dejourianaà Análise da Relação Prazer, Sofrimento e
Trabalho. São Paulo (SP): Atlas; 1994. p. 119-145.

25. Jorge IMP. Doenças Psicossomáticas Relacionadas ao Trabalho - Estudo de Caso. Florianópolis. Dissertação [Mestrado em Engenharia de Produção] Universidade Federal de Santa Catarina; 2004. [citado em 28 de outubro de 2017].Disponível em: https://repositorio.ufsc.br/bitstream/handle/ 123456789/101557/223668.pdf?sequence $=1$ \&isAllowed $=\mathrm{y}$.

26. Pereira JIM. Doenças Psicossomáticas Relacionadas ao Trabalho: estudo de caso. Florianópolis, 2004. 101f. Dissertação (Mestrado em Engenharia de Produção). Programa de Pós-Graduação em Engenharia de Produção. UFSC. Florianópolis, 2004.

27. Spector PE. Psicologia nas Organizações. São Paulo (SP): Saraiva; 2003.

28. Schlindwein VLDC. Histórias de Vida Marcadas por Humilhação, Assédio Moral e Adoecimento no Trabalho. Rev Psicologia \& Sociedade 2013; 25(2): 430439. [citado em 24 de novembro de 2017]. Disponível em: http://www.redalyc.org/pdf/3093/30932821 8020.pdf.

29. Bergamini GB, Aloise M. O Trabalho e - Seu Papel Social: Uma Perspectiva Existencial do Individuo Social. Porto Velho (RO): Netsaber; 2006. [citado em 24 de novembro de 2017]. Disponível em: http://artigos.netsaber.com.br/resumo_artig o_20060/artigo_sobre_o-trabalho-e-o-seupapel-social

30. Tartari JLO. Acidente de Trabalho. Rev. Jurídica da Universidade de Cuiabá 2016; 6(1): 75-79. [citado em 24 de novembro de 2017]. Disponível em: http://revistaemam.kinghost.net/revista/ind ex.php/rjunic/article/download/434/393. 


\section{Como citar (Vancouver)}

Silva JO, Ferreira SKA, Silva SF, Bergamini GB, Samuelsson E, Joner C et al. A correlação existente entre o estresse no ambiente de trabalho e doenças psicossomáticas. Rev Cient Fac Educ e Meio Ambiente [Internet]. 2017;8(2):177-191. DOI: http://dx.doi.org/10.31072/rcf.v8i2.552 